\title{
Neuroendovascular clinical trials disruptions due to COVID-19. Potential future challenges and opportunities
}

 \\ Aditya S Pandey $\left(10,{ }^{4}\right.$ Guilherme Dabus $\left(10,{ }^{5}\right.$ Ameer E Hassan (다, ${ }^{6}$ \\ Justin F Fraser (1) , ${ }^{7}$ Joshua A Hirsch (1) ${ }^{8}$ Rishi Gupta ${ }^{9}$ Ricardo Hanel (i) , ${ }^{10}$ \\ Albert J Yoo, ${ }^{11}$ Hormozd Bozorgchami, ${ }^{12}$ David Fiorella (1), ${ }^{13}$ J Mocco, ${ }^{14}$ \\ Adam S Arthur (10), ${ }^{15,16}$ Osama Zaidat 지, ${ }^{17}$ Adnan H Siddiqui (1) ${ }^{18}$
}

- Additional material is published online only. To view please visit the journal online (http://dx.doi.org/10.1136/ neurintsurg-2020-016502).

For numbered affiliations see end of article.

Correspondence to Dr Ansaar T Rai, Department of Interventional Neuroradiology, West Virginia University Rockefeller Neuroscience Institute, Morgantown, West Virginia, USA; ansaar.rai@gmail. com

Received 11 June 2020 Revised 17 June 2020 Accepted 20 June 2020 Published Online First 30 June 2020
Check for updates

(c) Author(s) (or their employer(s)) 2020. Re-use permitted under CC BY-NC. No commercial re-use. See rights and permissions. Published by BMJ.

To cite: Rai AT, LeslieMazwi TM, Fargen KM, et al. J Neurolntervent Surg 2020:12:831-835.

\section{ABSTRACT \\ OBJECTIVE}

To assess the impact of COVID-19 on neurovascular research and deal with the challenges imposed by the pandemic

Methods A survey-based study focused on randomized controlled trials (RCTs) and single-arm studies for acute ischemic stroke and cerebral aneurysms was developed by a group of senior neurointerventionalists and sent to sites identified through the clinical trials website (https://clinicaltrials.gov/), study sponsors, and physician investigators.

Results The survey was sent to 101 institutions, with 65 responding (64\%). Stroke RCTs were being conducted at $40(62 \%)$ sites, aneurysm RCTs at $22(34 \%)$ sites, stroke single-arm studies at 37 (57\%) sites, and aneurysm single-arm studies at $43(66 \%)$ sites. Following COVID-19, enrollment was suspended at 51 (78\%) sites-completely at 21 (32\%) and partially at 30 (46\%) sites. Missed trial-related clinics and imaging follow-ups and protocol deviations were reported by 27 (42\%), $24(37 \%)$, and $27(42 \%)$ sites, respectively. Negative reimbursements were reported at $17(26 \%)$ sites. The majority of sites, $49(75 \%)$, had put new trials on hold. Of the coordinators, 41 (63\%) worked from home and $20(31 \%)$ reported a personal financial impact. Remote consent was possible for some studies at 34 (52\%) sites and for all studies at $5(8 \%)$ sites. At sites with suspended trials $(n=51)$, endovascular treatment without enrollment occurred at 31 (61\%) sites for stroke and 23 (45\%) sites for aneurysms. A total of 277 patients with acute ischemic stroke and 184 with cerebral aneurysms were treated without consideration for trial enrollment. Conclusion Widespread disruption of neuroendovascular trials occurred because of COVID-19. As sites resume clinical research, steps to mitigate similar challenges in the future should be considered.

\section{INTRODUCTION}

The neurovascular community has had to date a relatively homogeneous response to the COVID-19 pandemic with regards to clinical care. Measures including elective case suspension and modifications for emergency treatment and safety procedures to accommodate patients with COVID-19 have been widely adopted. Societal guidelines containing overlapping recommendations, based on evidence in the literature and expert consensus, were published to codify this. ${ }^{1-3}$ By contrast, the impact of COVID-19 on clinical research efforts has been less clear.

The Food and Drug Administration (FDA) published its guidance on the 'Conduct Of Clinical Trials Of Medical Products During COVID-19 Pandemic' for the industry, investigators, and institutional review boards in March 2020 and updated these on April 2, 2020 (https://www.fda.gov/media/ 136238 /download). This guidance is expected to remain in effect until the public health emergency related to the pandemic is lifted by the Department of Health and Human Services. The FDA document is comprehensive and recognizes the challenges due to quarantines, suspension of trials, and interruption of supply chains. It also recognizes that protocol deviations are likely and that trial modifications may be required. Similar guidelines were issued by the European Medicines Agency on the management of clinical trials during the COVID-19 pandemic on April 28, 2020 (https://ec.europa.eu/ health/sites/health/files/files/eudralex/vol-10/guid anceclinicaltrials_covid19 en.pdf).

Disruption of clinical research infrastructure has implications for patient enrollment, study timing, sponsorship, and advances in clinical care and disease understanding. At the time of writing, fears of resurgent or re-emergent disease remain prominent. ${ }^{4}$ The impetus of this study was clinical trial suspension and challenges in patient monitoring experienced by several physician investigators and enrolling sites. This led to formulation of a hypothesis and study design which aimed to evaluate the impact of COVID-19 on neurovascular research studies and had a goal of better understanding the implications for existing and future trials.

\section{METHODS}

Institutional review board approval was not obtained as the survey did not involve study of human subjects. A survey writing group committee, comprising 12 neurointerventional physicians (four neuroradiologists, five neurosurgeons, and three 
neurologists) each with 10 or more years of experience in clinical practice, was assembled. All were involved in neurovascular clinical trials as site principal investigators and six were leading clinical trials as national principal investigators. The writing group was tasked with composing a concise survey, limited to fewer than 20 questions, to be completed by institutional research coordinators to explore the effect of COVID-19 on neurointerventional research and clinical trials (full survey can be found in online supplementary materials A). The survey focused on endovascular trials for acute ischemic stroke and cerebral aneurysms, including both randomized clinical trials (RCTs) and single-arm studies. The specific trial names or study sponsors were not recorded to protect confidentiality. The site name was only logged to assure one response from each site. No compensation was offered to participants. No requests to complete the survey were placed on public social media platforms. The questions were intended to explore key aspects of managing clinical trials in a restricted environment as imposed by the pandemic. These were identified by the writing group and fell into four categories: general disruption caused by trial suspensions and missed opportunities of enrollment, compromised trial quality due to inability of timely clinical and imaging follow-up, inability to enroll neurologically debilitated patients because legally authorized representatives were not at hand for face to face consent and dated remote consent procedures did not apply and, finally, personal effect of compensation or working conditions on study staff. The question about remote consent related to existing operating procedures for ongoing trials. The suspension of clinical trials was divided into partial or complete suspension based on whether some or all neuroendovascular trials were suspended. In cases of partial suspension, the question did not give details of the criteria for suspending some trials and not others. The survey also did not seek information on the size of the program, case volume, or association with a research consortium. This was partly to ensure that the brevity of the survey was maintained, and partly to maintain broad applicability of the survey regardless of program size, academic affiliation, or geographic location. The final questionnaire was also reviewed by three industry sponsors of clinical trials, two of which agreed to disclose their names (Stryker Neurovascular; Fremont, California, USA and Cerenovus; Miami, Florida, USA).

The survey sample was identified by creating a list of participants from the federal repository website (clinicaltrials.gov). The website allows combinations of search terms to identify clinical trials. The search terms used included 'stroke', 'thrombectomy', and 'brain aneurysms' with the geographic localizer of clinical trials in the 'United States'. The results were filtered for recruiting trials. The website provided contact details for each study. In a separate process, various study sponsors and principal investigators of ongoing clinical trials were approached by members of the writing group and asked to provide lists of participating sites. The accumulated sites were cross referenced with the list generated from clinicaltrials.gov to eliminate duplicates and when site information was missing, physician investigators localized by state were identified and invited individually through the Society of Neurointerventional Surgery senior physician membership (https://www.snisonline.org/). The final sample resulted in a total of 101 institutions identified for potential participation.

The finalized survey was prepared on Qualtrics (https://www. qualtrics.com) using an institutional license. The survey was distributed to the identified sites by email and was open for a 4-week period from April 21, 2020 to May 21, 2020. To boost the response rate, a digital link to the survey and a printable version (PDF, Word document) were emailed to the physician investigators and study coordinators midway through the 4-week period as a prompt. Surveys were completed voluntarily by a research coordinator at each institution with the help of the physician investigator as required. Each center was permitted only one response. The survey is presented as descriptive statistics.

\section{RESULTS}

The survey was sent to study coordinators at 101 institutions with 65 responding (64\%). All responding sites were actively involved in neurovascular trials before the COVID-19 pandemic. Of the returned forms, the only missing responses related to entry of the date of clinical trial suspension (if applicable). An evaluation of endovascular studies showed that a majority of sites were conducting stroke-related RCTs or single-arm studies. Most sites were also conducting single-arm aneurysm trials while a minority were participating in aneurysm-related RCTs (figure 1A).

During the pandemic, the majority of the sites either partially or completely suspended enrollment in clinical trials (figure 1B), with the suspension occurring between the middle to the end of March 2020. There was no correlation between site trial suspension and geographic location or academic affiliation. Suspension of sites by state showed a heterogeneity of response, with different sites in same state having different responses (figure 2). No correlation was found between trial suspension and the intensity of the pandemic.



Figure 1 (A) The randomized controlled trials (RCTs) and single arm studies for stroke and aneurysms being conducted at the responding sites $(n=65)$ prior to COVID-19. (B) clinical trial suspension at the responding sites during the pandemic. 


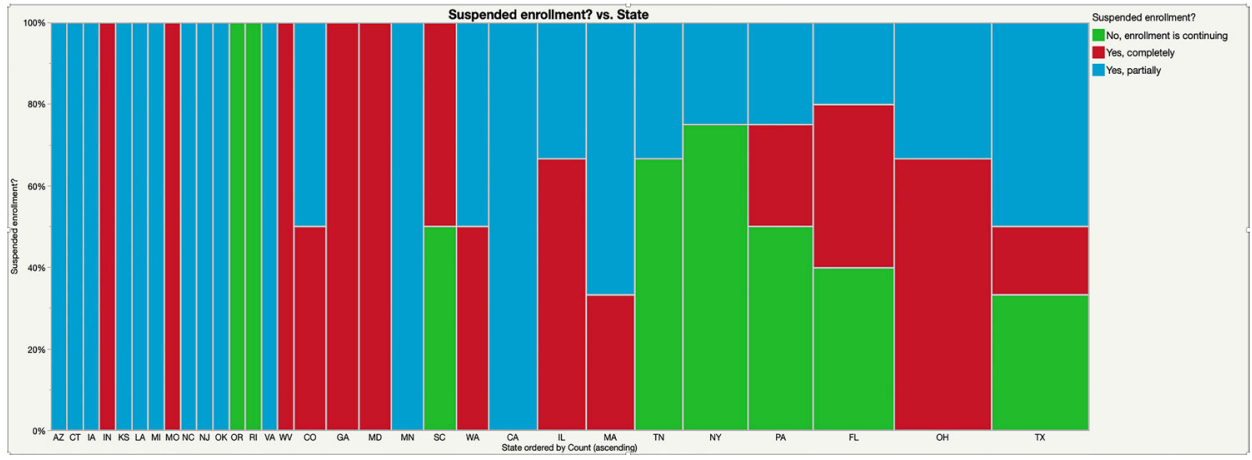

Figure 2 A chart of trial suspensions by state. The width of the bar for a given state correlates to the number of sites responding from that state. The wider the bar, the more the sites responding from that state. These are depicted in ascending order from left to right.

Endovascular stroke care continued in sites where enrollment had been suspended $(n=51)$, and most of these sites treated patients with acute ischemic stroke without enrollment. Almost half of the sites treated patients with an aneurysm (figure $3 \mathrm{~A}$ ). A total of 277 patients with endovascular stroke and 184 with endovascular aneurysm were treated without consideration for enrollment following suspension of clinical trials. Effects were also observed on patients already enrolled in trials. Forty-two percent of sites missed clinical follow-up visits, and 37\% missed imaging follow-up. Under half of the sites (42\%) reported protocol deviations because of the pandemic and a quarter reported loss of reimbursements possibly due to delayed or missed milestones or inability to enroll patients (figure $3 \mathrm{~B}$ ). Almost all $(61 / 65,94 \%)$ sites had developed alternative mechanisms such as telemedicine for clinical visits.

Most of the sites had received institutional review board guidance for the conduct of clinical trials $(n=57,88 \%)$ and most had received guidance from sponsors for either all $(n=31,48 \%)$ or some $(n=30,46 \%)$ of the clinical trials. Over half of the sites allowed electronic or phone consent for enrollment (figure 4). There were widespread challenges for study staff, with the majority of the study coordinators working from home $(n=41$, $63 \%)$ or a combination of home and office $(n=18,28 \%)$ and almost a third $(n=20,31 \%)$ reporting being personally affected by the pandemic either due to loss of pay, paid time off, or loss of benefits. The majority of the sites $(n=49,75 \%)$ had placed new trials on hold, with the number of postponed trials per site ranging from one to seven. Two sites reported a diagnosis of COVID-19 among the enrolled patients.

\section{DISCUSSION}

This study identified widespread disruptions to neurovascular clinical trial networks, most notably due to a suspension or postponement of trial enrollment, interruptions in scheduled patient follow-up, and challenges with study staff routines. Cessation of ongoing trials was nearly uniform, which may have downstream effects on trial validity and solvency. Understanding the effect of COVID-19 on clinical trials during the early months of the pandemic is especially important given the uncertainty about the future and potential need for subsequent lockdowns should widespread infection recur after resumption of elective procedures and relaxation of social distancing measures. One previous letter has been published, documenting decreased enrollment related to the pandemic in cancer-related clinical trials, ${ }^{5}$ and another systematic review on cancer research during COVID-19. ${ }^{6}$ The Federation of Italian Cooperative Oncology group issued a brief commentary and practice indications for drug studies. ${ }^{7}$ Other publications have included clinical trials for Alzheimer's research, ${ }^{8}$ and a review for guidance in head and neck research. ${ }^{9}$ At the time of writing, no published studies measuring the comprehensive impact of COVID-19 on research are available, and while this study focuses specifically on neurovascular research and trials, disruptions captured may well be generalizable to United States clinical trials in other medical specialties.

A prominent source of disruption identified by this survey was the complete or partial suspension of trial enrollment. Many sites with suspended enrollment treated potentially eligible patients with stroke and aneurysm. A total of 277 patients with

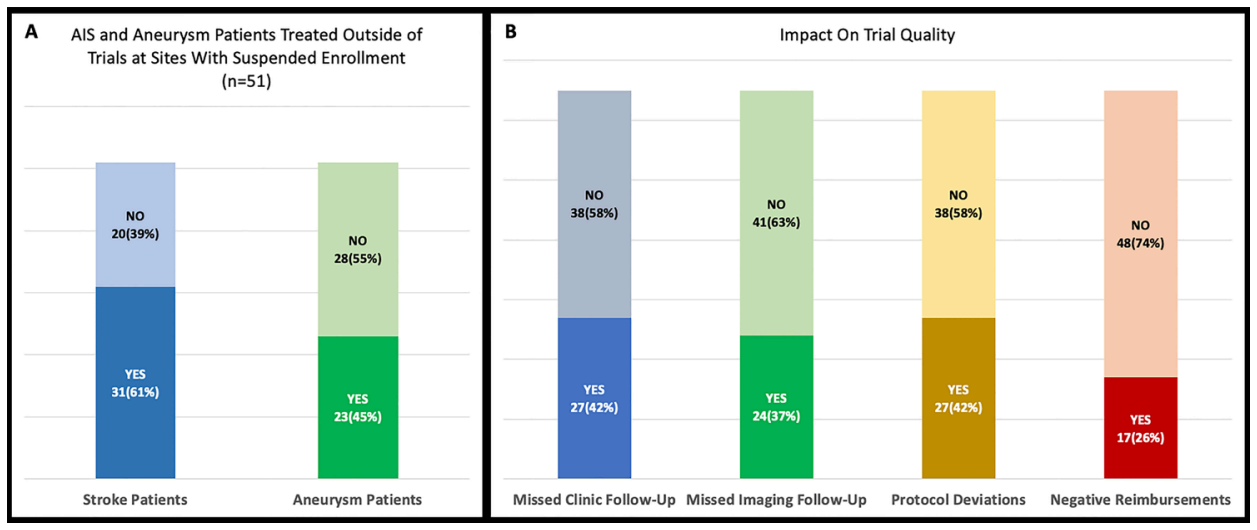

Figure 3 (A) Sites with partial or complete suspension $(n=51)$ reporting endovascular treatment of patients with stroke or an aneurysm without enrollment. (B) Sites reporting missed clinical or imaging follow-up visits, protocol deviations, and loss of reimbursements. AIS, acute ischemic stroke. 


\section{REMOTE CONSENTS}



Figure 4 Remote consent policies adopted at responding sites.

stroke and 184 with an aneurysm reportedly underwent endovascular therapy at these sites during the period in which the survey was conducted and thus could not be considered for enrollment owing to the suspension of clinical research. In view of the number of sites that did not respond to the survey, this number may be even higher. Unlike the 'clinical deficit', which is expected to be gradually overcome by returning case volume and case rescheduling, no similar option exists for research enrollment deficits. The most likely means of compensating for missed enrollments is to extend trial enrollment timelines. Such extension has implications for funding, compounded by potential contraction of funding following the wider economic impact of the global pandemic.

Beyond enrollment alone, trial quality was compromised. Many patients missed clinical and imaging follow-up milestones, and many sites reported protocol deviations due to the pandemic. These may increase as pandemic effects continue and could affect the veracity of overall trial data. Site trial infrastructure will have to adjust to new realities of reduced funding for many centers, with just over a quarter reporting a negative impact on reimbursements.

Human trial expertise has also been affected by the COVID-19 pandemic. Sites could experience reassignment, furloughing, or termination of study coordinators. One-third of responding coordinators reported a direct personal effect through loss of pay, time off, or benefits. As operations gradually return, this human expertise may need to be rebuilt. Trialists and staff will also need ongoing protection from potential infectious exposures. ${ }^{10}$ Failure to do so could affect a site's ability to perform existing and future trials unless alternative mechanisms are developed.

As clinical research activities resume with lifting of mandated lockdowns, this survey highlights several opportunities to update the conduct of clinical trials in accordance with the virtual care revolution brought about by the COVID-19 pandemic. $^{11} 12$ In this survey, $40 \%$ of the responding sites did not have any protocols in place for remote consent. Challenges to the traditional consent process could persist into the foreseeable future as hospital visitor restrictions are likely to remain in place for some time. Study sponsors, local institutional review boards, and federal authorities regulating these trials will have to demonstrate creativity in modernizing these procedures. Harnessing emerging virtual technologies that allow for face-to-face interactions with legally authorized representatives, electronic exchange of documents, and digital signatures, represents a clear opportunity. It is possible to comply with the ethical principles governing informed consent while updating these procedures to meet the current and future needs of operating with restricted visits. ${ }^{13}$

In the recovery phase there are calls in both public and medical circles for our healthcare infrastructure to focus on broadly improving our approaches, not simply returning to the prior state of normalcy. ${ }^{14}$ For acute trials in particular this could have broad positive implications for enrollment. Further, there are opportunities to enhance virtual capabilities to conduct trials, with reduced reliance on in-person monitoring and reporting forms. Based on existing data about telehealth, ${ }^{15}$ this may expand the trial footprint, decrease trial costs, and potentially increase trial efficiency. Trial reorganization into adaptive designs like platform trials may allow us to respond more rapidly to design changes and randomize more effectively as new questions arise. ${ }^{16}$ Early efforts are underway to develop a neurovascular platform trial infrastructure, and this should remain a focus for the neurointerventional community.

Another important consideration is how to handle potential trial candidates regarding COVID-19 exposure. In this survey, a small number (two sites) reported a positive COVID-19 diagnosis in enrolled patients. There is current uncertainty about how to process patients with COVID-19 in neurovascular trials or whether to screen based on symptoms or exposures. The direct effect of SARS-CoV-2 on cerebrovascular disease remains largely unknown, but early evidence suggests endothelial involvement ${ }^{17}$ and a range of syndromes ${ }^{18-20}$ that could confound the natural history of the disease or treatment response. This may be particularly relevant for studies of cerebrovascular conditions where patient outcomes may be directly influenced apart from the interventions being studied. How patients with COVID-19 should be handled by trials that are not focused on that disease specifically, is unknown. Testing all potential enrollees for COVID-19 and excluding all positive patients a priori seems reasonable, but this could result in unnecessary delays in enrollment or exclusion of patients who are COVID false positive or who have recovered from COVID. This larger question will probably be answered as we learn more about the virus. As more data are collected on the impact of the pandemic on clinical trials it may be possible to crystallize strategies for operating in a restrictive environment due to lingering concerns of outbreaks.

\section{Limitations}

This study has several important limitations. As a survey study, this study is subject to the inherent limitations of survey methodology, including recall and selection bias. The potential for selection bias is strong as those most affected by the pandemic may be more likely to complete the surveys. Additionally, centers with furloughed or terminated coordinators were unable to provide responses. A number of potentially important research or practice-specific questions, such as region or the prevalence of COVID-19 in the population, were not included in the survey by the writing group to improve participation rates. Questions were intentionally generated to assess a broad overview of research as opposed to specifically focus on individual trial statistics. The survey questions were purposely kept under 20, and therefore 
details that might have been helpful in further exploring some responses were not obtained. For example, the criteria for choosing to keep some trials open and others suspended are not known. Likewise, details of the causes of loss of trial-related reimbursements were not obtained. Additionally, implications for neurovascular basic or laboratory-based research, or for research in other domains, were similarly not assessed. The questions used were not previously validated and were designed specifically for the NI research coordinators involved in clinical trials, which may limit their generalizability to other specialties and make comparison with data from other surveys challenging. Lastly, since this type of pandemic has never occurred in modern history, there is no previously developed, validated survey instrument to study the effects of a global pandemic on research.

\section{CONCLUSION}

This is the first study comprehensively evaluating the effect of COVID-19 on clinical trials. Respondents indicated widespread disruption of neurovascular clinical research and research infrastructure during the COVID-19 pandemic. It has also revealed opportunities for increased infrastructural strength and pliability, including the increased use of virtual access for consent and follow-up, and the benefits of platform trial design at times when flexibility and responsiveness are key advantages. Many questions remain to be answered, including how we should incorporate COVID-19 screening into trial criteria. It should be expected that disruptions due to COVID-19 will continue, based on expert predictions of the disease course. Trial conduct in this restrictive environment will need to evolve. Decisions about how to maintain enrollment during the current pandemic and in the event of future similar disruptions ${ }^{21}$ must be prioritized.

\section{Author affiliations}

'Interventional Neuroradiology, West Virginia University Rockefeller Neuroscience Institute, Morgantown, West Virginia, USA

${ }^{2}$ Department of Neurosurgery, Massachusetts General Hospital, Boston, Massachusetts, USA

${ }^{3}$ Department of Neurological Surgery and Radiology, Wake Forest University, Winston-Salem, North Carolina, USA

${ }^{4}$ Department of Neurosurgery, University of Michigan, Ann Arbor, Michigan, USA

${ }^{5}$ Department of Interventional Neuroradiology and Neuroendovascular Surgery, Miami Neuroscience Institute and Miami Cardiac \& Vascular Institute - Baptist Hospital, Miami, Florida, USA

${ }^{6}$ Department of Neurology, University of Texas Rio Grande Valley School of Medicine, Edinburg, Texas, USA

${ }^{7}$ Department of Neurological Surgery, University of Kentucky, Lexington, Kentucky, USA

${ }^{8}$ NeuroEndovascular Program, Massachusetts General Hospital, Boston, Massachusetts, USA

${ }^{9}$ Department of Neurosurgery, WellStar Health System, Marietta, Georgia, USA

${ }^{10}$ Stroke and Cerebrovascular Center, Baptist Medical Center Jacksonville,

Jacksonville, Florida, USA

${ }^{11}$ Department of Neurointervention, Texas Stroke Institute, Plano, Texas, USA

${ }^{12}$ Oregon Health and Science University, Portland, Oregon, USA

${ }^{13}$ Department of Neurosurgery, Stony Brook University, Stony Brook, New York, USA

${ }^{14}$ The Mount Sinai Health System, New York, New York, USA

${ }^{15}$ Semmes-Murphey Neurologic and Spine Institute, Memphis, Tennessee, USA

${ }^{16}$ Department of Neurosurgery, University of Tennessee Health Science Center,

Memphis, Tennessee, USA

${ }^{17}$ Department of Neuroscience, St Vincent Mercy Hospital, Toledo, Ohio, USA

${ }^{18}$ Department of Neurosurgery, Gates Vascular Institute at Kaleida Health, Buffalo, New York, USA

Correction notice This article has been corrected since it was published Online First. The title was updated from 'Neuroendovascular clinical trials disruptions due to COVID-19 potential future challenges and opportunities' to 'Neuroendovascular Clinical Trials Disruptions Due To COVID-19. Potential Future Challenges and Opportunities

Twitter Ansaar T Rai @Ansaar_Rai, Joshua A Hirsch @JoshuaAHirsch and Adam S Arthur@AdamArthurMD
Acknowledgements The authors acknowledge the study coordinators who diligently manage neurovascular clinical trials. Without their hard work these clinical trials will not be successful.

Contributors ATR, AHS, ASP, AEH, GD, OZ, AJY, ASA, JM, and DF contributed to the study design. ATR, TML-M, KMF, ASP, AEH, GD, and JFF contributed to manuscript preparation. Everyone contributed to data collection and gave input into the study design and manuscript preparation.

Funding The authors have not declared a specific grant for this research from any funding agency in the public, commercial or not-for-profit sectors.

Competing interests None declared.

Patient consent for publication Not required.

Provenance and peer review Not commissioned; externally peer reviewed.

Data availability statement Data sharing not applicable as no datasets generated and/or analysed for this study. No data are available.

Open access This is an open access article distributed in accordance with the Creative Commons Attribution Non Commercial (CC BY-NC 4.0) license, which permits others to distribute, remix, adapt, build upon this work non-commercially, and license their derivative works on different terms, provided the original work is properly cited, appropriate credit is given, any changes made indicated, and the use is non-commercial. See: http://creativecommons.org/licenses/by-nc/4.0/.

\section{ORCID iDs}

Ansaar T Rai http://orcid.org/0000-0001-9864-4805

Thabele M Leslie-Mazwi http://orcid.org/0000-0002-4191-2466

Kyle M Fargen http://orcid.org/0000-0001-8979-1993

Aditya S Pandey http://orcid.org/0000-0003-0789-4273

Guilherme Dabus http://orcid.org/0000-0003-1582-6035

Ameer E Hassan http://orcid.org/0000-0002-7148-7616

Justin F Fraser http://orcid.org/0000-0002-5980-3989

Joshua A Hirsch http://orcid.org/0000-0002-9594-8798

Ricardo Hanel http://orcid.org/0000-0001-7195-5806

David Fiorella http://orcid.org/0000-0002-2677-8780

Adam S Arthur http://orcid.org/0000-0002-1536-1613

Osama Zaidat http://orcid.org/0000-0003-4881-4698

Adnan H Siddiqui http://orcid.org/0000-0002-9519-0059

\section{REFERENCES}

1 Fraser JF, Arthur AS, Chen M, et al. Society of Neurolnterventional Surgery recommendations for the care of emergent neurointerventional patients in the setting of COVID-19. J Neurointerv Surg 2020;12:539-41.

2 Pandey AS, Ringer AJ, Rai A, et al. Letter: considerations for performing emergent neurointerventional procedures in a COVID-19 environment. Neurosurgery 2020;5.

3 Leslie-Mazwi TM, Fargen KM, Levitt M, et al. Preserving access: a review of stroke thrombectomy during the COVID-19 pandemic. AJNR Am J Neuroradiol 2020. doi:10.3174/ajnr.A6606. [Epub ahead of print: 21 May 2020].

4 Anderson RM, Heesterbeek $\mathrm{H}$, Klinkenberg $\mathrm{D}$, et al. How will country-based mitigation measures influence the course of the COVID-19 epidemic? Lancet 2020;395:931-4.

5 Unger JM, Blanke CD, LeBlanc M, et al. Association of the coronavirus disease 2019 (COVID-19) outbreak with enrollment in cancer clinical trials. JAMA Netw Open 2020;3:e2010651.

6 Moujaess E, Kourie HR, Ghosn M. Cancer patients and research during COVID-19 pandemic: a systematic review of current evidence. Crit Rev Oncol Hematol 2020;150:102972.

7 Pinto C, Cagnazzo C. Indications regarding the management of interventional clinical trials with drugs during the current COVID-19 emergency in Italy. ESMO Open 2020;5:e000782

8 Weinberg MS, Patrick RE, Schwab NA, et al. Clinical trials and tribulations in the COVID-19 era. Am J Geriatr Psychiatry 2020. doi:10.1016/j.jagp.2020.05.016. [Epub ahead of print: 19 May 2020].

9 Singh AG, Chaturvedi P. Clinical trials during COVID-19. Head Neck 2020. [Epub ahead of print: 29 Apr 2020].

10 Ran L, Chen X, Wang Y, et al. Risk factors of healthcare workers with corona virus disease 2019: a retrospective cohort study in a designated hospital of Wuhan in China. Clin Infect Dis 2020.

11 Schwamm LH, Erskine A, Licurse A. A digital embrace to blunt the curve of COVID19 pandemic. NPJ Digit Med 2020;3:64.

12 Hollander JE, Carr BG. Virtually perfect? telemedicine for Covid-19. N Eng/ J Med 2020;382:1679-81

13 Rai AT, Frei D. A rationale and framework for seeking remote electronic or phone consent approval in endovascular stroke trials - special relevance in the COVID-19 environment and beyond. J Neurointerv Surg 2020;12:654-7.

14 Mukherjee S. What the coronavirus crisis reveals about American medicine. The New Yorker, 2020 
15 Donelan K, Barreto EA, Sossong S, et al. Patient and clinician experiences with telehealth for patient follow-up care. Am J Manag Care 2019;25:40-4.

16 Schiavone F, Bathia R, Letchemanan K, et al. This is a platform alteration: a trial management perspective on the operational aspects of adaptive and platform and umbrella protocols. Trials 2019;20:264.

17 Varga Z, Flammer AJ, Steiger $\mathrm{P}$, et al. Endothelial cell infection and endotheliitis in COVID-19. Lancet 2020;395:1417-8.
18 Beyrouti R, Adams ME, Benjamin L, et al. Characteristics of ischaemic stroke associated with COVID-19. J Neurol Neurosurg Psychiatry 2020. doi:10.1136/jnnp2020-323586. [Epub ahead of print: 30 Apr 2020].

19 Hess DC, Eldahshan W, Rutkowski E. COVID-19-related stroke. Trans/ Stroke Res 2020;11:322-5.

20 Oxley TJ, Mocco J, Majidi S, et al. Large-vessel stroke as a presenting feature of Covid-19 in the young. N Engl J Med 2020;382:e60.

21 Gates B. Innovation for pandemics. N Eng/ J Med 2018;378:2057-60. 